New windows on massive stars: asteroseismology, interferometry, and spectropolarimetry

Proceedings IAU Symposium No. 307, 2014

G. Meynet, C. Georgy, J. H. Groh 8 Ph. Stee, eds.

(C) International Astronomical Union 2015 doi:10.1017/S1743921314006474

\title{
Investigation of the brightest stars in the Cyg OB2 association
}

\author{
Olga Maryeva ${ }^{1}$ and Sergey Parfenov ${ }^{2}$ \\ ${ }^{1}$ Special Astrophysical Observatory of the Russian Academy of Sciences \\ email: olga.maryeva@gmail.com \\ ${ }^{2}$ Ural Federal University
}

\begin{abstract}
We present the results of investigation of most luminous stars belonging to the Cyg OB2 association using quantitative analysis of high-resolution spectra. Physical parameters derived using the CMFGEN and TLUSTY atmospheric codes allow us to estimate the mass and age of these stars.
\end{abstract}

Keywords. stars: early-type stars: atmospheres stars: winds, outflows stars: evolution

\section{Introduction}

Cygnus OB2 (Cyg OB2) stellar association, discovered by Munch and Morgan in 1953, is the current leader in number of massive stars among Galactic OB-associations. The interest of researchers in individual stars and the association as a whole is not fading. Numerous articles have investigated the stellar population of Cyg OB2, its interstellar extinction, and star-formation history.

\section{Modeling results}

E. L. Chentsov and V. G. Klochkova have performed studies of objects belonging to Cyg OB2 for several years using the Russian $6 \mathrm{~m}$ telescope of the Special Astrophysical Observatory (SAO RAS). Chentsov et al. (2013) published deep spectral description of high resolution spectra of 13 stars in Cyg OB2 and spectral variability of the star \#12. We decided to continue the studies and calculate models of these stars.

We used the TLUSTY code by Lanz \& Hubeny (2003) to model the atmospheres of the dwarfs (Cyg OB2 \#6, \#16, and \#21) and CMFGEN (Hillier \& Miller 1998) for calculation of the atmospheres of supergiants. The results of modeling of the dwarfs are listed in Table 1 and displayed in Figure 1.

As of now, eighteen Galactic O-stars are classified as Ofc (Walborn et al. 2010). Four of them are in Cyg OB2. We modeled the spectra of \#9 (O 4.5 Ifc) and \#11 (O 5.5 Ifc). Table 2 lists the model parameters while the surface abundances of $\mathrm{H}, \mathrm{He}$, and CNO are given in Table 3 .

Table 1. The resulted parameters and their standard deviations obtained for dwarf stars considered in this study.

\begin{tabular}{l|c|c|c|c|c|c|c|c|c}
\hline & $\begin{array}{c}\text { Spec. } \\
\text { class }\end{array}$ & $\begin{array}{c}T_{\text {eff }} \\
\mathrm{kK}\end{array}$ & $\begin{array}{c}\log g \\
\mathrm{~cm} \mathrm{~s}^{-2}\end{array}$ & $\mathrm{He} / \mathrm{H}$ & $\begin{array}{c}V \sin i \\
\mathrm{~km} \mathrm{~s}^{-1}\end{array}$ & $\begin{array}{c}V_{\mathrm{micr}} \\
\mathrm{km} \mathrm{s}^{-1}\end{array}$ & $\begin{array}{c}M_{\star} \\
M_{\odot}\end{array}$ & $\log \left(L_{\star} / L_{\odot}\right)$ & $\begin{array}{c}\text { Age } \\
\mathrm{Myr}\end{array}$ \\
\hline 6 & O8 V V & 32.8 & 3.72 & 0.11 & 210 & 12 & $24 \pm 5$ & $5.1 \pm 0.3$ & $5.6 \pm 1.3$ \\
16 & O7.5 V & 33.3 & 3.78 & 0.16 & 200 & 12 & $24 \pm 5$ & $5.1 \pm 0.3$ & $5.2 \pm 1.4$ \\
21 & B0 V & 31.2 & 3.97 & 0.12 & 25 & 9 & $18 \pm 2$ & $4.7 \pm 0.2$ & $5.1 \pm 1.6$
\end{tabular}



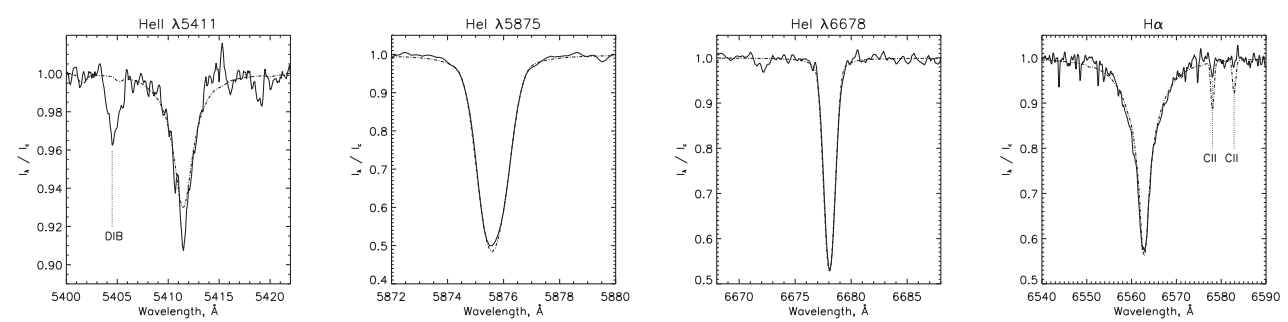

Figure 1. Comparison of the observed spectrum of Cyg OB2 \#21 with the best-fit models.

Table 2. Atmospheric parameters of Cyg OB2 \#9 and Cyg OB2 \#11.

\begin{tabular}{|c|c|c|c|c|c|c|c|c|c|c|c|}
\hline & $\begin{array}{l}T_{\star} \\
\mathrm{kK}\end{array}$ & $\begin{array}{l}R_{\star} \\
R_{\odot}\end{array}$ & $\begin{array}{l}T_{\text {eff }} \\
\mathrm{kK}\end{array}$ & $\begin{array}{c}R_{2 / 3} \\
R_{\odot}\end{array}$ & $\mid \begin{array}{c}L_{\star} \\
10^{5} L_{\odot}\end{array}$ & $\mid \begin{array}{c}\dot{M}_{\mathrm{uncl}} \\
10^{-6} M_{\odot} \mathrm{yr}^{-1}\end{array}$ & $10^{-6}$ & $\begin{array}{l}\dot{M}_{\mathrm{cl}} \\
M_{\odot} \mathrm{yr}^{-1}\end{array}$ & $f_{\infty}$ & $\mid \begin{array}{c}V_{\infty} \\
\mathrm{km} \mathrm{s}^{-1}\end{array}$ & $\beta$ \\
\hline$\# 9$ & 37 & 20 & 36 & 21 & 6.5 & 3.9 & & 1.1 & 0.08 & 1500 & 1.3 \\
\hline$\# 11$ & 35 & 22 & 34.4 & 22.7 & 6.5 & 6 & & 1.7 & 0.08 & 2200 & 1.3 \\
\hline
\end{tabular}

Table 3. The abundances of chemical elements. Solar values are from Asplund et al. (2009).

\begin{tabular}{llll}
\hline Element & Cyg OB2 \#9 & Cyg OB2 \#11 & Sun \\
\hline $\mathrm{H}$ & 12 & 12 & 12 \\
$\mathrm{He}$ & $10.7 \pm 0.15$ & $10.85 \pm 0.15$ & $10.93 \pm 0.01$ \\
$\mathrm{C}$ & $8.08 \pm 0.05$ & $8.5 \pm 0.09$ & $8.39 \pm 0.05$ \\
$\mathrm{~N}$ & $8.37 \pm 0.03$ & $8.28 \pm 0.03$ & $7.78 \pm 0.06$ \\
$\mathrm{O}$ & $8.18 \pm 0.07$ & $8.17 \pm 0.07$ & $8.66 \pm 0.05$
\end{tabular}

We found $\mathrm{He} / \mathrm{H} \approx 0.1$ in the atmospheres of the Ofc stars. Within the errors, it means that their He abundance is equal to initial He abundance of the environment and that $\mathrm{He} / \mathrm{H}$ did not change during the lifetimes of Cyg OB2 \#9 and \#11. In Cyg OB2 \#11, the $\mathrm{N}$ abundance is lower than in other "normal" $\mathrm{O}$ stars, while the $\mathrm{C}$ abundance is solar. In Cyg OB2 \#9 the fraction of $\mathrm{N}$ is higher than in \#11 and the fraction of $\mathrm{C}$ is lower. Cyg OB2 \#9 is closer to "normal" O stars than \#11. Probably, there is no mixing in atmospheres of Ofc stars that transports the products of CNO cycle from the core towards the stellar surface.

The results discussed here have been published in: Maryeva et al. (2014), Maryeva et al. (2013) and Maryeva \& Zhuchkov (2012).

Olga Maryeva thanks the International Astronomical Union and Dynasty Foundation for grants. The study was supported by the Russian Foundation for Basic Research (projects no. 14-02-31247,14-02-00291).

\section{References}

Asplund, M., Grevesse, N., Sauval, A. J., \& Scott, P. 2009, ARAEGA 47, 481

Chentsov, E. L., Klochkova, V. G., Panchuk, V. E., Yushkin, M. V., \& Nasonov, D. S. 2013, Astronomy Reports 57, 527

Hillier, D. J. \& Miller, D. L. 1998, ApJ 496, 407

Lanz, T. \& Hubeny, I. 2003, ApJS 146, 417

Maryeva, O., Zhuchkov, R., \& Malogolovets, E. 2014, Proc. ASA 31, 20

Maryeva, O. V., Klochkova, V. G., \& Chentsov, E. L. 2013, Astrophysical Bulletin 68, 87

Maryeva, O. V. \& Zhuchkov, R. Y. 2012, Astrophysics 55, 371

Walborn, N. R., Sota, A., Maíz Apellániz, J., et al. 2010, ApJ (Letters) 711, L143 